\title{
APPLICATION OF THE MODIFIED MAGNETOELASTIC METHOD AND AN ANALYSIS OF THE MAGNETIC FIELD
}

\author{
Tomáš Klier ${ }^{a}$, Tomáš MíčKa ${ }^{a}$, Michal Polák ${ }^{b, *}$, Tomáš PlachÝ ${ }^{b}$, \\ Milan Hedbávn'́ ${ }^{c}$, Roman JelíneK ${ }^{c}$, Filip Bláha ${ }^{b, d}$ \\ a Pontex, spol. s r. o., Bezová 1658, 14714 Prague 4, Czech Republic \\ ${ }^{b}$ Department of Mechanics, Faculty of Civil Engineering, Czech Technical University in Prague, Thakurova 7, \\ 16629 Prague 6, Czech Republic \\ ${ }^{c}$ Freyssinet CZ a. s., Zápy 267, 25001 Brandýs nad Labem, Czech Republic \\ $d$ Department of Concrete and Masonry Structures, Faculty of Civil Engineering, Czech Technical University in \\ Prague, Thákurova 7, 16629 Prague 6, Czech Republic \\ * corresponding author: polak@fsv.cvut.cz
}

\begin{abstract}
In the technical praxis there is very often a need of axial force determination in the important structural elements of a building during its construction or operational state. The paper presents practical application of the new approach based on the magnetoelastic principle, especially aimed on experimental evaluation of the axial forces in the prestressed reinforcement on prestressed concrete structures. Described approach is usable not only for newly built structures but in particular for existing ones. The experiment was realized on a prestressed concrete beam dismantled from a bridge that was put out of operation. The influence of a simulated defect of the reinforcement on its magnetoelastic properties has also been investigated. During result evaluation, the knowledge based on the theoretical analysis of the electromagnetic field was used.
\end{abstract}

KEYwords: Concrete, tensile force, magnetoelastic method, prestressed strand, prestressed cable, prestressed wire, elastomagnetic sensor.

\section{INTRODUCTION}

In civil engineering practice, five experimental techniques are usually used for evaluation of axial tensile forces, the direct measurement of the force by a preinstalled load cell, the approach based on a strain measurement with strain gauges, the vibration frequency method [1, 2], the force determination in a flexible structural element based on the relation between the transverse force and the caused transverse displacement [3], 4, and the magnetoelastic method [5] 10. All mentioned methods have their advantages and disadvantages that are discussed in more detail in the reference [7] and [8. Only three of them are usable for new experiment on an existing structure that is in service.

The first one is the vibration frequency method that is applicable only to structural elements with relatively long free vibrating length. The uncertainty of the specified force is moreover significantly influenced by the element bending stiffness and by the element boundary conditions especially if they are complicated and vague [1, 2].

The second one is method based on the relation between the transverse force and the transverse element displacement that is usable only for significantly flexible elements with a relatively long free length. Due to the transverse displacement the tensile force in the considerably short element during the experiment substantially increases even in the range of tens of percent [3, 4].

The third one is new approach based on the magnetoelastic method [5-77]. According to our opinion, the new approach is the most suitable procedure for tensile stress evaluation in prestressed elements on existing structures that are in service.

The basic aim of the project is to research and develop a new approach based on the "magnetoelastic" physical principle. The approach is designed for the measurement of axial forces in structural elements made of ferromagnetic materials (in steel prestressed rods, wires and cables) on existing or newly constructed building structures. The research solved by our team seeks to focus primarily on solving the task of "determining the actual size of the force in a prestressed concrete element on an existing structure" which is very common in technical practice but is often not resolvable by any other method used nowadays.

This paper presents one of the typical problems that have been solved so far [8 10]. In the conclusion, the properties and risks of this approach are summarized.

\section{THEORY - PHYSICAL PRINCIPLE}

Elastomagnetic resp. magnetoelastic principle was explained in more detail in the sources [5, 6. Here are briefly summarized the basic facts:

- The mechanical state and magnetic properties of the ferromagnetic material interact. 
- The magnetic properties of the particular material define the relationship between the magnetic field shape described by the magnetic field intensity " $\mathrm{H}$ " $\left[\right.$ A. $\left.\mathrm{m}^{-1}\right]$ and the shape of the magnetic induction field "B" $[\mathrm{T}]$ (also named the magnetic flux density). For non-ferromagnetic materials the dependence is elementary, while the dependence for ferromagnetic ones is very complex, influenced by a number of parameters (temperature, mechanical stress, history of magnetic and mechanical stress for example).

- The magnetic field intensity "H" can be monitored locally, for example, by Hall sensors. The field of magnetic induction "B" may be monitored by means of a coil that integrates the normal component of the magnetic induction field over the area of individual coil turns.

\section{THEORY - PHYSICAL PRINCIPLE}

Figure 1 shows a diagram of a fully equipped elastomagnetic sensor (hereinafter EM sensor). Fundamental sensor components are a controlled magnetic field source (the primary coil in this case), a sensor of magnetic field intensity " $\mathrm{H}$ " in a measured crosssection (the system of Hall's sensors, the secondary coil 2), a sensor of magnetic flux that is closely related to the magnetic induction " $\mathrm{B}$ " in the measured section (the secondary coil 1) and EM sensor protection against magnetic influences from its surroundings (steel shield).

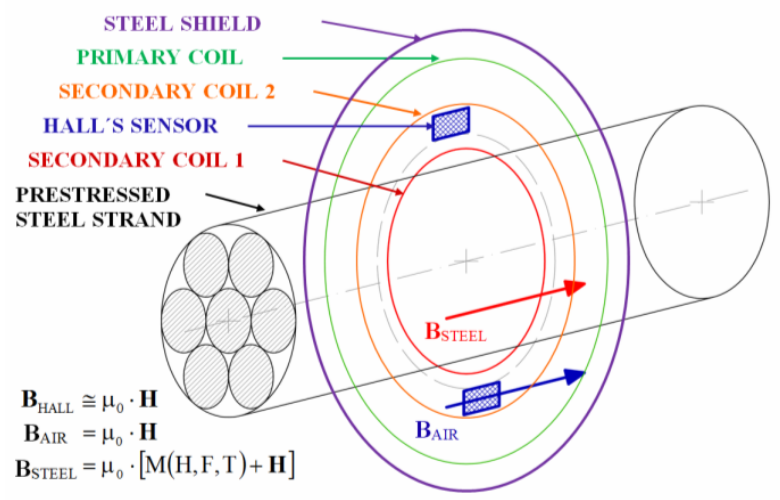

Figure 1. Diagram of a fully equipped elastomagnetic sensor (EM sensor).

The function and principle of Hall's sensors were explained in more detail in the reference [6].

\subsection{Maximal COnfiguration}

The reader of this paper certainly assumes that a fully featured sensor offers the greatest possibilities to increase accuracy and reduce uncertainties in determining the axial force in the measured prestressed element. But the reader will also not be surprised that the fully equipped sensor is spatially larger, that may limit its applicability, and also more complicated. This complexity affects its higher production time, the higher usage requirements for this sensor in civil engineering practise and as well as the higher demands on the equipment of the used measuring system.

\subsection{Minimal COnfiguration}

Here is described an opposite extreme of the EM sensor - the minimally featured one. The minimalist EM sensor consists of a primary coil and a secondary coil 1 only. In this case, the intensity of the magnetic field " $\mathrm{H}$ " is determined indirectly from a completely different physical quantity, from the current flows through the primary coil. The greatest risk of using such sensor results from this approach. Any change in the magnetic surroundings around the sensor (a removal of a massive steel falsework after concrete hardening for example) causes completely "silently" the substantial or fatal changes in sensor parameters. The sensor's steel shielding reduces the range of this effect, the level of this reduction depends on the quality of the EM sensor shielding, nevertheless it is never one hundred percent. For example, the minimum sensor configuration is completely unusable when applied to steel-fibre concrete.

The EM sensors and their appropriate equipment that are standardly used at present [8-10] evaluate measured prestressed forces in a relatively simple way. However, these standard EM sensors, that have hitherto been used in civil engineering practice, are composed from the primary and secondary coils only and that is the minimal possible configuration of the EM sensor as it is mentioned in this chapter.

\subsection{MeAsurement OF MAGNetiC FIELD INTENSITY}

If we are not satisfied with the indirect measurement of the magnetic field intensity " $\mathrm{H}$ " described in the previous paragraph, it is possible to use the Hall sensors for this purpose. Well-calibrated Hall sensors allow very accurate determination of " $\mathrm{H}$ " in close proximity to the studied object, for example even in the gaps between the strands or between the prestressed wires in the experimentally investigated prestressed cable, and they are particularly advantageous when the observed magnetic field is asymmetrical. One disadvantage of this EM sensor configuration is the fact that modern Hall sensor are integrated semiconductor circuits that are not, in principle, reliable for long-term use in exposed surroundings.

An alternative to Hall sensors is to add a secondary coil 2 to the sensor construction. This coil, as well as the concentrically located secondary coil 1 , observes a change in the magnetic flow passing through its turns. The mutual difference between magnetic flows measured by the coil 2 and the coil 1 indicates a change in flow passing through the annulus between turns of both secondary coils. In this space there are only non-ferromagnetic materials and therefore the magnetic flux can be readily recalculated to the average normal component of the magnetic field intensity " $\mathrm{H}$ " 
in this annulus. The disadvantage of this sensor arrangement is that the intensity of the magnetic field is determined in comparison with the Hall sensors at a greater distance from the studied prestressed elements and also that the secondary coil 2 increases the dimension of the sensor transverse cross-section. On the other hand, the advantage of this EM sensor configuration is that the output of the EM sensor is integrated from the larger area and therefore it is more resistant to the effects of local anomalies in the magnetic field and the application of secondary coil 2 also allows to not use the Hall probes within the EM sensor and it positively affects the reliability of the EM sensor in its long-term use.

\subsection{Optimal COnfiguration of the EM SENSOR FOR EXISTING STRUCTURES}

The so far gained experience by EM sensor applications on existing structures shows that it seems optimal to assemble an EM sensor from all the parts shown in Figure 1. Its complete configuration allows the best available calibration of an EM sensor under specific conditions. If the long-term use of the retrofitted EM sensors is assumed, then it is possible to dismantle the Hall probes from the EM sensor after its initial calibration, and then process only the outputs of a pair of secondary coils. Otherwise, for a one-off experiment, it is possible to omit the secondary coil 2 installation because there is no use for it.

\section{EXPERIMENT ON A REAL STRUCTURE}

The experiment was carried out on the prefabricated prestressed concrete beam of the type KA-67, which was dismantled from a bridge put out of service and which did not serve the original purpose for years. Thanks to this fact, the experiment could be done without minimizing damage of the concrete part of the beam. It was also possible to carry out test in which an influence of a simulated defect of the studied cable on its magnetoelastic properties has been investigated. The force in the prestressed steel cable was intentionally released by means of the gradual cut off prestressed wires forming cable.

This experiment was focused on the following problematic areas: verification of real spatial requirements for an EM sensor additional installation on a prestressed cable, optimization of the geometric arrangement of the EM sensor to real conditions and theoretical modelling of the electromagnetic field of the sensor taking into account the general geometric arrangement of the measured cross-section of the studied prestressed reinforcement. The theoretical model created in the software Ansys Maxwell 3D that corresponds to the experiment arrangement and conditions is shown in Figure 5

The design of the applied EM sensor has been optimized for the particular conditions of one-off experiment realized on the "small" in-situ cable, see

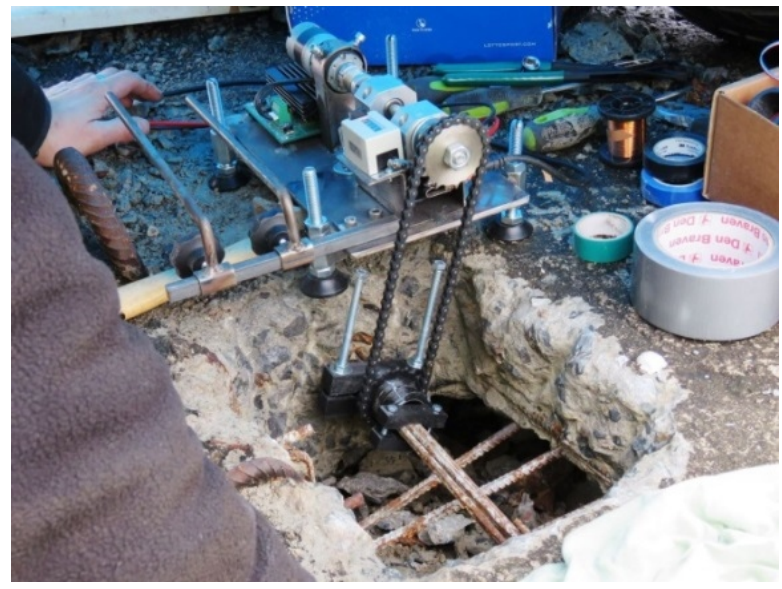

Figure 2. The production of the secondary coil in-situ.

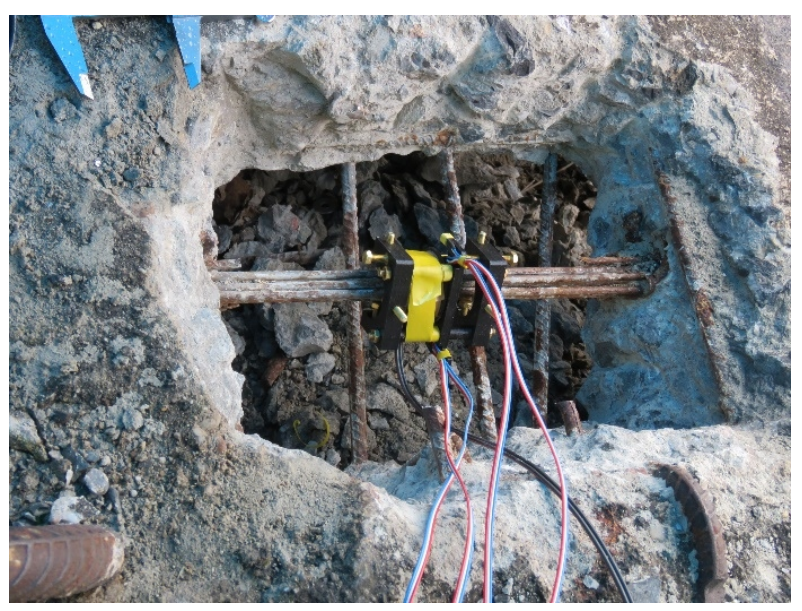

FiguRE 3. The holder for the set of four Hall sensors with the embedded secondary coil 1 positioned on the studied cable.

Figure 2 A cable composed of only a few prestressed wires allowed the use of a portable primary coil to emit a sufficiently intense electromagnetic field, see Figure 2 2 Only the secondary coil 1 was produced in-situ, see Figure 1 and Figure 3. The set of Hall sensors located on the holder stabilizing the ideal mutual position of all sensor parts relative to each other was also demountable and portable. The steel shield with regard to detailed magnetic field monitoring in the measured cable cross-section by four Hall probes was not necessary.

The condition of successful application of the elastomagnetic measuring method is the detailed knowledge of the composition of the electromagnetic field generated by the primary coil in the measured cross-section of the studied prestressed element. Based on the analysis of the results obtained from the theoretical model (see Figure 3), it is possible to create a functional relationship between the real measured variables and the variables necessary for the evaluation of the solved prestressing force or the stress in the studied prestressed reinforcement. For example, we need to know the average value of the magnetic field intensity in the 


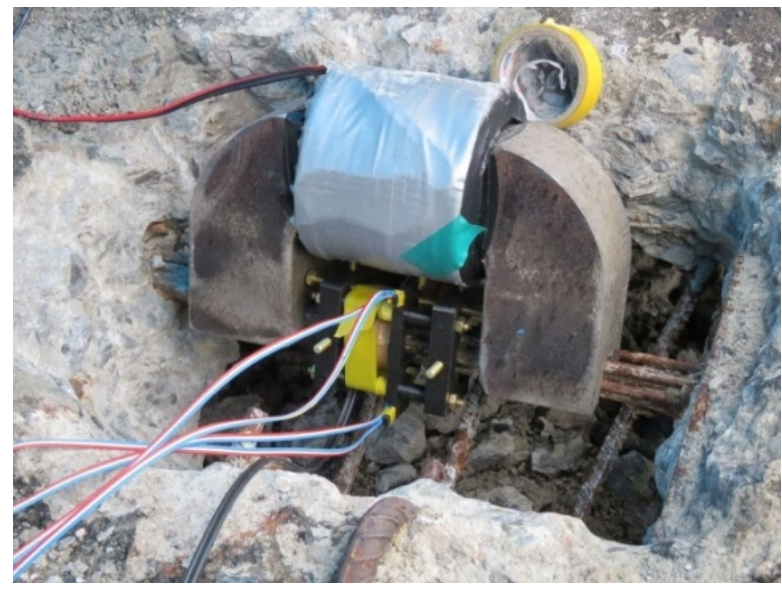

Figure 4. The complete EM sensor on the cable.

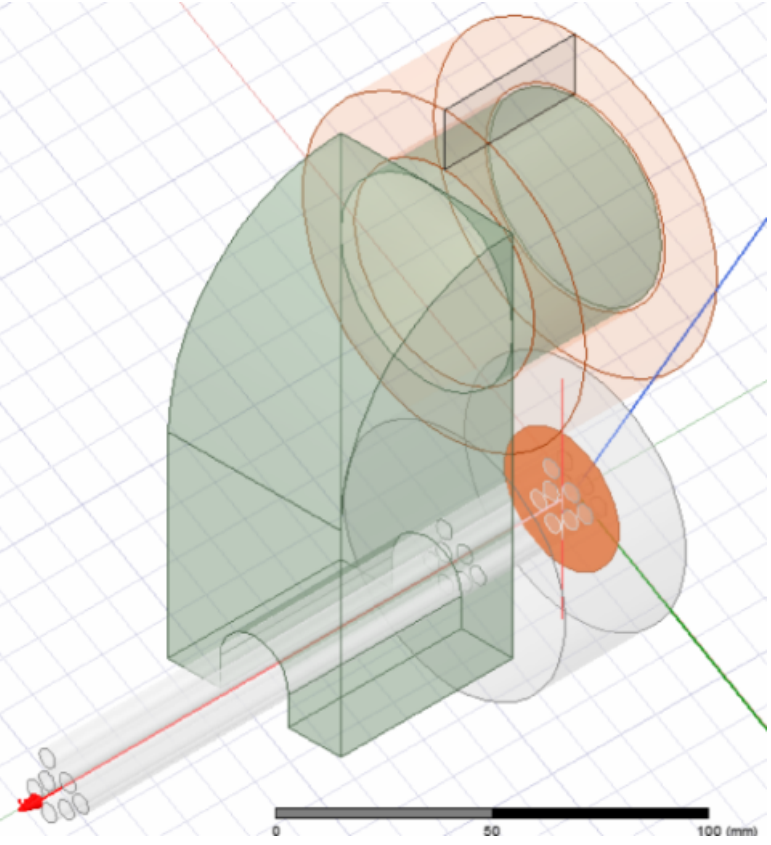

Figure 5. The theoretical model of the EM sensor positioned on the studied prestressed cable - only half of the model visualization.

cross-section of the studied prestressed element, but we can only measure it locally at the positions of Hall sensors.

In Figure 7 there is a graphical representation of the results obtained during the realized experiment. The red lines with the "+" node symbols show the measured values of the average stress in the cable. They differ by the evaluation curve used for determination of the results. $\mathrm{D}_{\text {avg }}$ is the mean curve obtained from available results evaluated by previously realized experiments carried out for three different types of prestressed wires [6, 7] and $\mathrm{D}_{1}$ is the most appropriate evaluation curve obtained for the prestressed wire most similar to that used in the tested structural element.

The black and blue curves show the computed values of the average stress in the studied cable according to different computational models of the prestressed

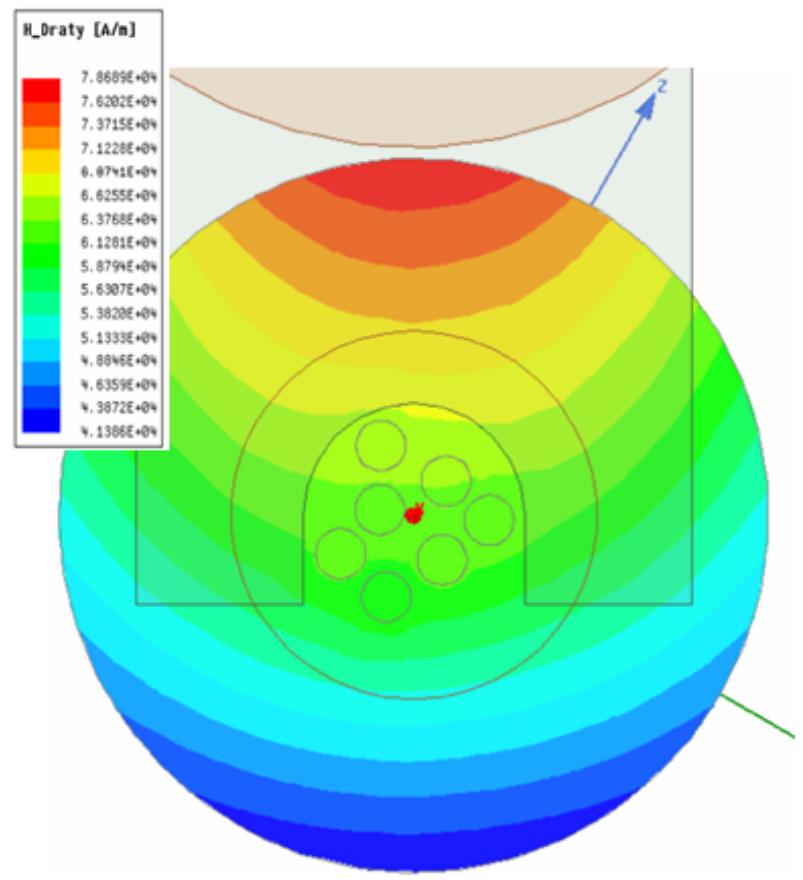

FiguRE 6. The theoretical model of the EM sensor with the studied prestressed cable - magnetic field intensity in the cross-section of the secondary coil 1.

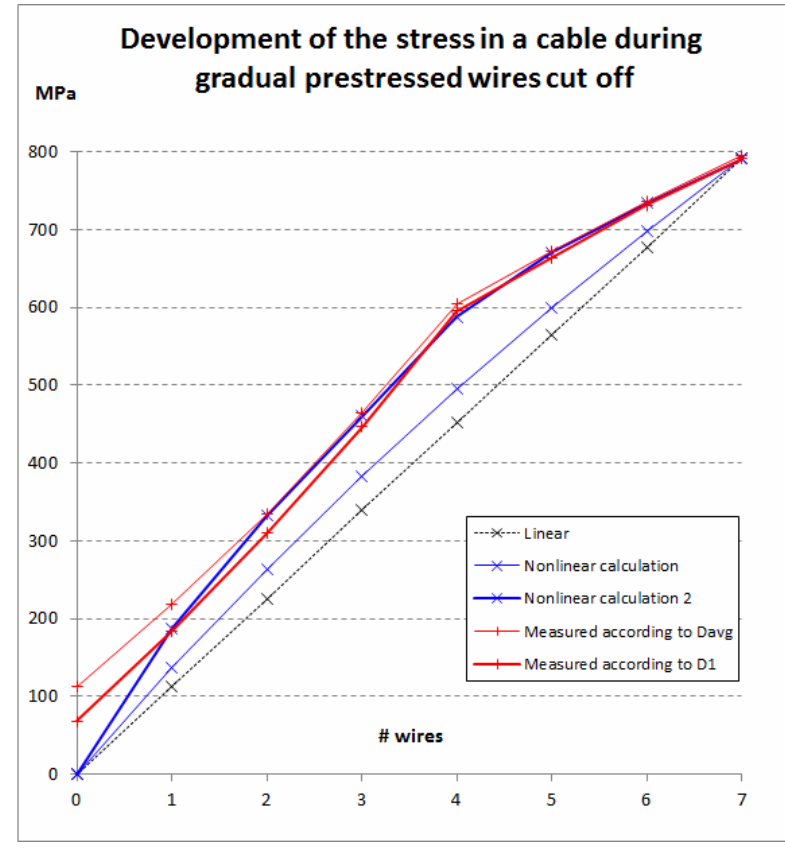

Figure 7. 7 Development of the axial tensile force in the cable during the gradual prestressed wires cut off.

concrete beam. The black curve corresponds to a model where zero deformation of concrete is assumed during the gradual cutting off of the wires. The blue thin curve corresponds to the assumption of uniform elastic deformation in concrete. In addition, the blue thick curve includes a local change in the field of deformation due to the demolition work performed to allow the installation of the EM sensor. 
The very good match between the average stresses measured and evaluated according to the curve D1 (thick red curve in Figure 7) and the non-linear static model of the observed structural element (thick blue curve in Figure 7) is obvious. The more significant differences in these two curves occurred only in the area where almost all cable wires are cut off. The deviation between these two curves is probably caused by different stresses than axial. In the case of the described experiment, it is probably due to the weight of the primary coil acting in the transverse direction on the remaining uncut wires.

\section{Conclusions}

The presented experiment confirmed the applicability of the developed new measuring approach based on the "magnetoelastic" physical principle to real existing structures. Accuracy and uncertainty of results obtained by this approach are significantly affected, as other methods, by the quality of the experiment realization, but also by the quality of the used evaluation curve. Obviously, the highest quality of the evaluation curve can be obtained from a prestressed reinforcement sample taken directly from the investigated structure, but in most real cases it is difficult or totally impossible. Therefore, it is important to gradually create the widest possible database of evaluation curves for prestressed elements commonly used in the building industry, both today and in the past.

\section{ACKNOWLEDGEMENTS}

The results presented in this paper are outputs of the research project FV 30457 "Utilization of a Magnetoelastic Method for Increasing the Reliability and Durability of Existing and Newly Built Prestressed Concrete Structures" supported by Ministry of Industry and Trade of the Czech Republic.

\section{REFERENCES}

[1] M. Polák, T. Plachý. Determination of forces in roof cables at administrative center amazon court. Procedia Engineering 48:578-582, 2012.

[2] M. Polák, T. Plachý. Experimental evaluation of tensile forces in short steel rods. Applied Mechanics and Materials 732:333-336, 2015.
[3] P. Fajman, M. Polák. Measurement of structural cable of membranes. In Proceedings of the 50th Annual Conference on Experimental Stress Analysis, vol. 50, pp. 61-64. 2012.

[4] P. Fajman, M. Polák, J. Máca, T. Plachý. The experimental observation of the prestress forces in the structural elements of a tension fabric structure. Applied Mechanics and Materials 486:189-194, 2014.

[5] T. Klier, T. Míčka, M. Polák, et al. Modern method for measurement of the axial forces in elements of building structures. In Proceedings of the conference Betonářské dny 2016, vol. 23. ČBS, 2016.

[6] T. Klier, T. Míčka, T. Plachý, et al. The verification of a new approach to the experimental estimation of tensile forces in prestressed structural elements by method based on the magnetoelastic principle. MATEC Web of Conferences 107(00015):1-6, 2017.

[7] T. Klier, T. Míčka, M. Polák, et al. The in situ application of a new approach to experimental estimation of tensile forces in prestressed structural elements by method based on the magnetoelastic principle. In Proceedings of the 55th Conference on Experimental Stress Analysis 2017, vol. 55, pp. 122-132. Technical University of Košice, 2017.

[8] A. M. Sarmento, A. Lage, E. Caetano, J. Figueiras. Stress measurement and material defect detection in steel strands by magneto elastic effect. comparison with other non-destructive measurement techniques. In Proceedings of the 6th International Conference on Bridge Maintenance, Safety and Management IABMAS 2012, pp. 914-921. IABMAS, 2012.

[9] M. Chandoga, P. Fabo, A. Jaroševič. Measurement of forces in the cable stays of the apollo bridge. In Proceedings of the 2nd fib Congress, pp. 674-675. fib, 2006.

[10] H.-J. Wichmann, A. Holst, H. Budelmann. Magnetoelastic stress measurement and material defect detection in prestressed tendons using coil sensors. In Proceedings of rth International Symposium on NonDestructive Testing in Civil Engineering NDTCES̆O9, p. 6. Laboratoire Central des Ponts et Chausses, 2009. 